Pacific Journal of Mathematic 


\title{
SYMMETRY AND NONSYMMETRY IN THE GROUP ALGEBRAS OF DISCRETE GROUPS
}

\author{
JoE W. JENKINS
}

\begin{abstract}
A Banach *-algebra $\mathscr{C}$, with identity $e$, is symmetric if $x x^{*}+e$ is regular for each $x$ in $\mathscr{C}$. In this paper we generalize certain conditions on a discrete group $G$ that are known to be sufficient to ensure symmetry of $\ell_{1}(G)$. Also we define semi-symmetry and derive an inequality that must be satisfied if $\ell_{1}(G)$ is not semi-symmetric. Finally we show that if a group contains a free subsemigroup on two or more generators then $\ell_{1}(G)$ is not symmetric.
\end{abstract}

Let $G$ be a discrete group. $\ell_{1}(G)$ the group algebra of $G$. $\ell_{1}(G)$ is a Banach *-algebra with involution defined pointwise by $x^{*}(g)=$ $\overline{x\left(g^{-1}\right)}$ and with convolution as multiplication. The mapping $g \rightarrow \delta_{g^{\prime}}$ where $\delta_{g}(s)=0$ if $s \neq g$ and $\delta_{g}(g)=1$, is a homomorphism of $G$ into $\ell_{1}(G)$. In general, we will not distinguish between $g$ and $\delta_{g}$. Note that if $x \in \ell_{1}(G)$ then $x$ can be written in the form $x=\sum_{g \in G} x(g) g$.

$\mathscr{H}(G)$ (or $\mathscr{H}$ ) will denote the real linear subspace of hermitian elements of $\ell_{1}(G) . \quad \mathscr{C}_{f}(G)$ will be the subspace of $\mathscr{C}(G)$ consisting of all elements $x$ such that

$$
N(x)=\{g \mid x(g) \neq 0\}
$$

is finite.

Let $\mathscr{C}$ denote the natural cone in $\ell_{1}(G)$, i.e., $\mathscr{K}$ is the cone generated by all elements of the form $x x^{*}$ where $x \in \ell_{1}(G)$. Denote by $\mathscr{F}_{0}(\mathscr{H})$ the continuous linear functionals defined on $\mathscr{H}$, nonnegative on $\mathscr{K} \cap \mathscr{H}$ and one at the identity.

The right regular representation of $\ell_{1}(G)$ over $\ell_{2}(G), x \rightarrow R_{x}$, is defined by: $R_{x}(y)=y x$, for each $y \in \ell_{2}(G)$.

DeFinition 1.1. $\ell_{1}(G)$ is semi-symmetric if $x \in \mathscr{X}_{f}(G)$ and $\mathrm{sp}\left(R_{x}\right) \geqq$ 0 imply $(x+e)^{-1} \in \ell_{1}(G)$.

LEMMA 1.2. If $\ell_{1}(G)$ is semi-symmetric then

(i) $\operatorname{sp}\left(x x^{*}\right) \geqq 0$ for each $x$ in $\ell_{1}(G)$ with $N(x)$ finite, and

(ii) if $x \in \mathscr{H}_{f}(G)$ then $\mathrm{sp}(x)$ is real.

The proof of this lemma is essentially a duplication of the proof of the corresponding results for an arbitrary symmetric Banach *-algebra. 
Let $P_{f}(G)$ be the subset of $\mathscr{C}_{f}(G)$ consisting of all elements with nonnegative spectra. We observe that $\lambda x \in P_{f}(G)$ if $\lambda>0$ and $x \in P_{f}(G)$, and that, since $\ell_{1}(G)$ is semi-simple,

$$
P_{f}(G) \cap-P_{f}(G)=\{0\} .
$$

Lemma 1.3. If $\ell_{1}(G)$ is semi-symmetric then $P_{f}(G)$ is a cone.

Proof. We need only show that $x+y \in P_{f}(G)$ if $x \in P_{f}(G)$ and $y \in P_{f}(G)$. Let $x \in P_{f}(G)$ and $y \in P_{f}(G)$. Then $\operatorname{sp}\left(R_{x}\right) \geqq 0$ and $\operatorname{sp}\left(R_{y}\right) \geqq$ 0 . Thus $R_{x}$ and $R_{y}$ are positive definite operators on $\ell_{2}(G)$ and hence also $R_{x}+R_{y}=R_{x+y}$. Therefore $\operatorname{sp}\left(R_{x+y}\right) \geqq 0$ and thus

$$
(x+y+e)^{-1} \in \ell_{1}(G) \text {. }
$$

If $\alpha>0$ then $\alpha^{-1} x$ and $\alpha^{-1} y$ are in $P_{f}(G)$. Hence

$$
\left(e+\alpha^{-1} x+\alpha^{-1} y\right)^{-1}=\alpha(\alpha e+x+y)^{-1} \in \ell_{1}(G) .
$$

Therefore $-\alpha \notin \mathrm{sp}(x+y)$ and, since $\mathrm{sp}(x+y)$ is real, $\mathrm{sp}(x+y) \geqq 0$.

If $\ell_{1}(G)$ is symmetric, then for each $x \in \mathscr{\mathscr { C }}(G)$

$$
\operatorname{sp}(x) \subset\left\{f(x) \mid f \in \mathscr{F}_{0}(\mathscr{H})\right\} \text {. }
$$

(This result is implicit in the usual proof of Raikov's Theorem, see [7]). If $\ell_{1}(G)$ is semi-symmetric an abbreviated version of this result can be proven.

Lemma 1.4. If $\ell_{1}(G)$ is semi-symmetric and $x \in \mathscr{C}_{f}(G)$ then

$$
\operatorname{sp}(x) \subset\left\{f(x) \mid f \in \mathscr{F}_{0}(\mathscr{X})\right\} \text {. }
$$

Proof. Let $x \in \mathscr{X}_{f}(G)$ be given. Denote by $\mathscr{C}(x)$ a maximal commutative *-subalgebra of $\ell_{1}(G)$ containing $x$, and by $\Delta(\mathscr{L}(x))$ the Gelfand representations of $\mathscr{C}(x)$. It is well known that if $y \in \mathscr{C}(x)$ then

$$
\operatorname{sp}_{\mathscr{\varkappa}(x)}(y)=\mathrm{sp}_{\ell_{1}(G)}(y)(=\mathrm{sp}(y)) .
$$

Since $\ell_{1}(G)$ is semi-symmetric, $P_{f}(G)$ is a cone. Hence, if we set

$$
\mathscr{C l}_{f}(x)=\mathscr{C l}(x) \cap \mathscr{X}_{f}(G)
$$

then $P_{f}(G)$ induces an order on $\mathscr{C l}_{f}(x)$. Furthermore, if for $\delta \in A(\mathscr{C}(x))$ we set $\delta_{f}=\delta_{i \mathscr{N}(x)}$, then $\delta_{f}$ is positive with respect to this order. By the Monotone Extension Theorem, $\delta_{f}$ has a positive extension to $\mathscr{H}_{f}(G)$ if

$$
\left(y+\mathscr{C l}_{f}(x)\right) \cap P_{f}(G) \neq \varnothing
$$


is equivalent with

$$
\left(y+\mathscr{L}_{f}(x)\right) \cap-P_{f}(G) \neq \varnothing
$$

for each $y \in \mathscr{K}_{f}(G)$.

Assume $z \in\left(y+\mathscr{L}_{f}(x)\right) \cap P_{f}(g)$. Then there is a $z^{\prime} \in \mathscr{l}_{f}(x)$ such that $y+z^{\prime} \in P_{f}(G)$. Hence

$$
\operatorname{sp}\left(y+z^{\prime}\right) \subset[0, a]
$$

for some $a>0$. Let $z^{\prime \prime}=z^{\prime}-a e$, then $z^{\prime \prime} \in \mathscr{l l}_{f}(x)$ and

$$
\mathrm{sp}\left(y+z^{\prime \prime}\right)=\mathrm{sp}\left(y+z^{\prime}-a e\right)=\mathrm{sp}\left(y+z^{\prime}\right)-a \subset[-a, 0] .
$$

Thus

$$
\left(y+\mathscr{L}_{f}(x)\right) \cap-P_{f}(G) \neq \varnothing .
$$

A similar argument establishes the converse.

Let $\bar{\delta}_{f}$ be an extension of $\delta_{f}$ given by the preceding argument. If $y \in \mathscr{Y C}_{f}(G)$ then $y-\nu(y) e \in-P_{f}(G)$. Hence $\bar{\delta}_{f}(y-\nu(y) e) \leqq 0$. But

$$
\bar{\delta}_{f}(y-\nu(y) e)=\bar{\delta}_{f}(y)-\nu(y) .
$$

Thus $\bar{\delta}_{f}(y) \leqq \nu(y)$. Similarly, $\bar{\delta}_{f}(y) \geqq-\nu(y)$. Therefore

$$
\left|\bar{\delta}_{f}(y)\right| \leqq \nu(y) \leqq\|y\|
$$

for each $y \in \mathscr{Y}_{f}(G)$. Since $\mathscr{Y}_{f}(G)$ is dense $\mathscr{Y}, \bar{\delta}_{f}$ has a continuous extension, $f_{\tilde{o}}$, to $\mathscr{Y}$. Since the closure of $P_{f}(G)$ contains the natural cone $\mathscr{K}, f_{i} \in \mathscr{F}_{0}(\mathscr{H})$.

Now, if $x \in \mathscr{X}_{f}(G)$ and $\alpha \in \mathrm{sp}(x)$ then there exist an $\mathscr{C}(x)$ and $\delta \in \Delta(. \mathscr{C}(x))$ such that $\delta(x)=\alpha$. But then $f_{\delta}(x)=\delta(x)=\alpha$. Hence

$$
\operatorname{sp}(x) \subset\left\{f(x) \mid f \in \mathscr{F}_{0}(\mathscr{C})\right\} .
$$

It is natural to ask how symmetry of $\ell_{1}(G)$ and semi-symmetry of $\ell_{1}(G)$ are related. The following theorems provide a partial answer.

THEOREM 1.5. Assume that $\ell_{1}(G)$ is semi-symmetric and that whenever $\lim _{n} x_{n}=x$ for $\left\{x_{n}\right\} \subset \mathscr{H}_{f}(G), \lim _{n} \nu\left(x_{n}\right)=\nu(x)$; then $\iota_{1}(G)$ is symmetric.

Proof: Let $x \in \ell_{1}(G)$ be given and select $\left\{x_{n}\right\} \subset \mathscr{K}_{f}(G)$ such that $\lim _{n} x_{n} x_{n}^{*}=x x^{*}$. Then, $\lim _{n} \nu\left(x_{n} x_{n}^{*}\right)=\nu\left(x x^{*}\right)$. Hence, if $\varepsilon>0$ is given, there is a $k$ such that

$$
\nu\left(x_{k} x_{k}^{*}\right)>\nu\left(x x^{*}\right)-\varepsilon / 2
$$

and 


$$
\left\|x_{k} x_{k}^{*}-x x^{*}\right\|<\varepsilon / 2 \text {. }
$$

But, by Lemma 1.4, there is an $f_{0} \in \mathscr{F}_{0}(\mathscr{H})$ such that

$$
f_{0}\left(x_{k} x_{k}^{*}\right)=\nu\left(x_{k} x_{k}^{*}\right) \text {. }
$$

Since each $f \in \mathscr{F}_{0}(\mathscr{H})$ has $\|f\|=1$,

$$
f\left(x_{k} x_{k}^{*}-x x^{*}\right)=f\left(x_{k} x_{k}^{*}\right)-f\left(x x^{*}\right)<\varepsilon / 2 .
$$

Thus

$$
f_{0}\left(x x^{*}\right)>f_{0}\left(x_{k} x_{k}^{*}\right)-\varepsilon / 2=\nu\left(x_{k} x_{k}^{*}\right)-\varepsilon / 2>\nu\left(x x^{*}\right)-\varepsilon .
$$

Hence

$$
\sup _{f \in x^{-}(x)} f\left(x x^{*}\right)=\nu\left(x x^{*}\right)
$$

for each $x \in \ell_{1}(g)$, and hence $\ell_{1}(G)$ is symmetric.

If $\ell_{1}(G)$ is symmetric then, by Raikov's theorem, (c.f. [8]), the spectral radius of each element of the form $x x^{*}$ is equal $\left\|T_{x x^{*}}\right\|$ for some *-representation $x \rightarrow T_{x}$. However, this *-representation need not be the right regular representation over $\iota_{2}(G)$. If we assume $G$ is amenable, then this latter representation weakly contains all other *-representations, ([6]), and hence the spectral radius of $x x^{*}$ is given by $\left\|R_{x x^{*}}\right\|$. Using these facts we can prove

THEOREM 1.6. If $\ell_{1}(G)$ is symmetric and if $G$ is amenable then $\iota_{1}(G)$ is semi-symmetric.

Proof. Suppose that $x \in \mathscr{K}_{f}(G),-1 \in \mathrm{sp}(x)$ and $\operatorname{sp}\left(R_{x}\right)$ is nonnegative. Let $y=x-\nu\left(R_{x}\right) e$ then

$$
-1-\nu\left(R_{x}\right) \in \operatorname{sp}(y)
$$

and

$$
\operatorname{sp}\left(R_{y}\right)=\operatorname{sp}\left(R_{x}-\nu\left(R_{x}\right) e\right) \subset\left[-\nu\left(R_{x}\right), 0\right] .
$$

Therefore

$$
\nu\left(y y^{*}\right)=\nu\left(y^{2}\right) \geqq\left(1+\nu\left(R_{x}\right)\right)^{2},
$$

and

$$
\nu\left(R_{y y^{*}}\right)=\nu\left(R_{y}^{2}\right) \leqq \nu\left(R_{x}\right)^{2}
$$

But

$$
\nu\left(R_{y y^{*}}\right)=\left\|R_{y y^{*}}\right\| .
$$

and, since $G$ is amenable, 


$$
\left\|R_{y y^{*}}\right\| \geqq\left\|T_{y y^{*}}\right\|
$$

for any ${ }^{*}$-representation $z \rightarrow T_{z}$. Therefore

$$
\nu\left(y y^{*}\right)=\left[1+\nu\left(R_{x}\right)\right]^{2}>\nu\left(R_{x}\right)^{2} \geqq\left\|R_{y y^{*}}\right\| \geqq\left\|T_{y y^{*}}\right\|
$$

for any *-representation $z \rightarrow T_{z}$. This contradicts Raikov's criteria for symmetry. Hence, if $\mathrm{sp}\left(R_{x}\right)$ is nonnegative, then $-1 \notin \mathrm{sp}(x)$.

REMARK. The dual hypothesus at Theorem 1.6, namely, that $\ell_{1}(G)$ was symmetric and that $G$ was amenable, was necessary. Although all known pertinent results tend to indicate that symmetry of $\ell_{1}(G)$ implies amenability of $G$, we do not know this to be true.

2. A sufficient condition for semi-symmetry. If $H$ is a subgroup of $G$ then there is a cannonical embedding of $\ell_{1}(H)$ into $\ell_{1}(G)$. We will not distinguish between an element of $\ell_{1}(H)$ and its image in $\ell_{1}(G)$. Since for each $x \in \ell_{1}(H), \mathrm{sp}_{\ell_{1}(H)}(x)=\mathrm{sp}_{\ell_{1}(G)}(x)$ (cf. [3]), we are assured that this laxity will cause no confusion when making spectral considerations.

Let $m(G)$ be the space of bounded functions defined on $G$. The mapping $\theta \rightarrow \theta^{v}$, where

$$
\theta^{v}(x)=\sum_{g \in G} \theta(g) x(g)
$$

for $x \in \ell_{1}(G)$, is an isometric isomorphism of $m(G)$ onto $\ell_{1}(G)^{*}$.

For $A \subset G$, let $\langle A\rangle$ be the group generated by $A$.

Lemma 2.1. Let $x \in \ell_{1}(G)$. Then $x$ has no left inverse if, and only if, there is a $\theta \in m(\langle N(x)\rangle)$ such that $\|\theta\|=1=\theta(e)$ and the null space of $\theta^{v}$ contains the left ideal generated by $x$.

Proof. Assume $x$ has no left inverse in $\ell_{1}(G)$. The preceding remarks imply that $x$ has no left inverse in $\ell_{1}(\langle N(x)\rangle)$.

Let $L$ be the left ideal in $\ell_{1}(\langle N(x)\rangle)$ generated by $x$. Now, if $y \in \ell_{1}(\langle N(x)\rangle)$ and $\|y\|<1$, then

$$
(e+y)^{-1}=e+\sum_{n=1}^{\infty}(-1)^{n} y^{n}
$$

is in $\ell_{1}(\langle N(x)\rangle)$. Hence, if $\|e-z\|<1$, set $y=-e+z$, and then $z^{-1}=(e+y)^{-1}$ is in $\ell_{1}(\langle N(x)\rangle)$. Thus

$$
L \cap\left\{y \in \ell_{1}(\langle N(x)\rangle) \mid\|e-y\|<1\right\}=\varnothing,
$$

and the distance of $L$ from $e$ is at least one. Hence the desired $\theta^{v}$ exist. 
The converse is obvious.

Let $x \in \mathscr{\mathscr { C }}_{f}(G)$ such that $x+e$ is singular and $\mathrm{sp}\left(R_{x}\right)$ is nonnegative. Let $A=N(x) \cup\{e\}, H=\langle A\rangle$ and $\mathrm{s}(n)=c\left(A^{n}\right)$, the cardinality of $A^{n}$. Enumerate the elements of $H$ in the following manner:

$$
\left\{g_{1}, g_{2}, \cdots, g_{s(1)}\right\}=A
$$

and

$$
\left\{g_{s(n)+1}, \cdots, g_{s(n+1)}\right\}=A^{n+1} \sim A^{n}
$$

for $n=1,2, \cdots$.

Since $x+e$ is singular, and $x+e$ is hermitian, $x+e$ has neither a right nor a left inverse. Hence there is a $\theta \in m(H)$ such that $\theta(e)=1=\|\theta\|$ and the null space of $\theta^{v}$ contains $L$, the left ideal generated by $x+e$. For $\theta^{v}$ to vanish on all $L$, it is necessary and sufficient that, in particular, $\theta^{v}\left(g_{i} x\right)=0$ for each $g_{i} \in H$.

Let $\theta_{i}=\theta\left(g_{i}\right)$ for $i=1,2, \cdots$, and for each positive integer $n$, define $\theta(n+1)$ in $\iota_{2}(H)$ by

$$
\theta(n+1)\left(g_{i}\right)=\left\{\begin{array}{l}
\theta_{i}, \text { if } 1 \leqq i \leqq s(n+1) \\
0, \text { if } i>s(n+1)
\end{array} .\right.
$$

Then

$$
\left\|R_{x+e}(\theta(n+1))\right\|_{2}^{2} \geqq\|\theta(n+1)\|_{2}^{2}+2\left(R_{x}(\theta(n+1)), \theta(n+1)\right) .
$$

But $\operatorname{sp}\left(R_{x}\right) \geqq 0$, hence

$$
\left(R_{x}(\theta(n+1)), \theta(n+1)\right) \geqq 0 .
$$

Therefore

2.2

$$
\left\|R_{x+e}(\theta(n+1))\right\|_{3}^{2} \geqq\|\theta(n+1)\|_{2}^{2}
$$

for $n=1,2, \cdots$.

Now, if $g_{i} \in A^{n}$ then

$$
N\left(g_{i}(x+e)\right) \subset A^{n} A=A^{n+1} .
$$

Thus

$$
\begin{aligned}
R_{x+e}(\theta(n+1))\left(g_{i}\right) & =[\theta(n+1)(x+e)]\left(g_{i}\right) \\
& =\sum_{j=1}^{\infty}\left[\theta(n+1)\left(g_{i}\right)\right]\left[(x+e)\left(g_{j}^{-1} g_{i}\right)\right] \\
& =\sum_{\substack{j=1 \\
s(n+1)}} \theta_{j}\left[(x+e)\left(g_{i}^{-1} g_{j}\right)\right] \\
& =\sum_{j=1}^{s(n+1)} \theta_{j}\left[g_{i}(x+e)\left(g_{j}\right)\right] \\
& =\theta^{v}\left(g_{i}(x+e)\right)=0 .
\end{aligned}
$$


If $g_{i} \in H \sim A^{n+2}$ then

$$
N(g(x+e)) \subset H \sim A^{n+1} .
$$

Hence again

$$
R_{x+e}(\theta(n+1))\left(g_{i}\right)=0 .
$$

Therefore

$$
\left\|R_{x+e}(\theta(n+1))\right\|_{2}^{2}=\sum_{j=s(n)+1}^{s(n+2)}\left|R_{x+e}(\theta(n+1))\left(g_{j}\right)\right|^{2} .
$$

But for $g_{j} \in A^{n+2} \sim A^{n}$,

$$
N\left(g_{j}(x+e)\right) \subset A^{n+3} \sim A^{n-1} .
$$

Hence

$$
\begin{aligned}
& \left|R_{x+e}(\theta(n+1))\left(g_{j}\right)\right|^{2} \\
\leqq & \left(\sum_{i=s(n-1)+1}^{s(n+1)}\left|\theta_{i}\left[g_{j}(x+e)\left(g_{i}\right)\right]\right|\right)^{2} \\
\leqq & \left(\sum_{i=s(n-1)+1}^{s(n+1)}\left|g_{j}(x+e)\left(g_{i}\right)\right|^{2}\right)\left(\sum_{i=s(n-1)+1}^{s(n+1)}\left|d_{j i} \theta_{i}\right|^{2}\right) \\
\leqq & \|x+e\|_{i=s}^{2} \sum_{i=s(n-1)+1}^{s(n+1)}\left|d_{j i} \theta_{i}\right|^{2}
\end{aligned}
$$

where $d_{j i}=0$ if $g_{j}(x+e)\left(g_{i}\right)=0$ and one otherwise. Note that for fixed $j, d_{j i} \neq 0$ for at most $c(A) i$ 's. Therefore

$$
\begin{aligned}
\left\|R_{x+e}(\theta(n+1))\right\|_{2}^{2} & \leqq \sum_{j=s(n)+1}^{s(n+2)}\left(\|x+e\|_{i=s(n-1)+1}^{2} \mid \sum_{j i}\left(\left.\theta_{i}\right|^{2}\right)\right. \\
& \leqq c(A)\|x+e\|_{i=s(n-1)+1}^{2} \sum_{i(n+1)}^{s(n+1)}\left|\theta_{i}\right|^{2} .
\end{aligned}
$$

We also have

$$
\|\theta(n+1)\|_{2}^{2}=\sum_{j=1}^{s i n+1)}\left|\theta_{j}\right|^{2} .
$$

Combining these results by 2.2 . we have

$$
c(A)\|x+e\|_{2}^{2} \sum_{j=s(n-1)+1}^{s(n+1)}\left|\theta_{j}\right|^{2} \geqq \sum_{j=1}^{s(n+1)}\left|\theta_{j}\right|^{2}
$$

for each $n=2,3, \cdots$.

We compile the above argument in

THEOREM 2.4. If $\ell_{1}(G)$ is not semi-symmetric then for some $x \in \mathscr{Y}_{f}(G)$ there is a $\theta=\left(\theta_{i}\right) \in m(\langle N(x)\rangle)$ such that $\|\theta\|=1=\theta(e)$ and $\left(\theta_{i}\right)$ satisfies 2.3 . 
3. Condition $S S$. For a given $G$ let $\mathscr{S}(G)$ denote the family of finite symmetric subsets of $G$ containing the identity. Adel 'sonVel' skii and Šreider, [1], considered the following condition on a group $G$ :

$(A-S)$ for each $A \in \mathscr{S}(G)$

$$
c\left(A^{n}\right)=o\left(d^{n}\right) \text { for any } d>1 .
$$

They proved that if $G$ satisfies $(A-S)$ then $G$ is amenable. Hulanicki, [5], later showed that if a group satisfies $(A-S)$ then $x x^{*}+e$ is regular for each $x$ in $\ell_{1}(G)$ with finite support.

We now define a condition which is weaker than $(A-S)$ :

(SS) for each $A \in \mathscr{S}(G)$

$$
\lim \inf _{n} c\left(A^{n+1} \sim A^{n}\right)^{1 / n} \leqq 1 .
$$

It is not difficult to show that if $G$ satisfies $(A-S)$ then $G$ satisfies $(S S)$ and that if $G$ satisfies $(S S), G$ is amenable. We also have

THEOREM 3.1. If $G$ satisfies $S S$ then $\ell_{1}(G)$ is semi-symmetric.

Proof. If $\ell_{1}(G)$ is not semi-symmetric then by Theorem 2.4 there is a $\theta=\left(\theta_{i}\right) \in m(\langle A\rangle)$, where $A=N(x) \cup\{e\}$, such that $\|\theta\|=$ $1=\theta(e)$ and

$$
c(A)\|x+e\|_{2}^{2} \sum_{j=s(n-1)+1}^{s(n+1)}\left|\theta_{j}\right|^{2} \geqq \sum_{j=1}^{s(n+1)}\left|\theta_{j}\right|^{2} \geqq \sum_{j=1}^{s(n-1)}\left|\theta_{j}\right|^{2}
$$

for each $n=2,3, \cdots$. Let $a^{\prime}=c(A)\|x+e\|_{2}^{2}, a=\left(a^{\prime}+1\right) / a^{\prime}$, and

$$
b=\sum_{j=1}^{s(2)}\left|\theta_{j}\right|^{2} .
$$

Then, since $\theta(e)=1$ and $e \in A, b>0$. We have

$$
\sum_{j=1}^{s(4)}\left|\theta_{j}\right|^{2}=\sum_{j=1}^{s(2)}\left|\theta_{j}\right|^{2}+\sum_{j=s(2)+1}^{s(4)}\left|\theta_{j}\right|^{2} \geqq a b ;
$$

and if

$$
\sum_{i=1}^{s(2 n)}\left|\theta_{j}\right|^{2} \geqq(a)^{n-1} b
$$

then

$$
\sum_{j=1}^{s(2 n+2)}\left|\theta_{j}\right|^{2}=\sum_{j=1}^{s(2 n)}\left|\theta_{j}\right|^{2}+\sum_{j=s(2 n)+1}^{s(2 n+2)}\left|\theta_{j}\right|^{2} \geqq(a)^{n-1} b+\left(1 / a^{\prime}\right)\left[(a)^{n-1} b\right] \geqq(a)^{n} b .
$$

Therefore 


$$
\sum_{j=1}^{s(2 n+2)}\left|\theta_{j}\right|^{2} \geqq(a)^{n} b
$$

for $n=2,3, \cdots$.

Since $\|\theta\|=1 ;\left|\theta_{j}\right| \leqq 1$ for each $j$. Hence

$$
\sum_{j=s(2 n)+1}^{s(2 n+2)}\left|\theta_{j}\right|^{2} \leqq c\left(A^{2 n+2} \sim A^{2 n}\right) .
$$

Consequently

$$
c\left(A^{2 n+2} \sim A^{2 n}\right) \geqq\left(1 / a^{\prime}\right) a^{n} b .
$$

for $n=2,3, \cdots$ If $B=A^{2}$ then $B \in \mathscr{S}(G)$ and $\liminf _{n} C\left(B^{n+1} \sim B^{n}\right)>1$. This contradiction implies $\ell_{1}(G)$ is semi-symmetric

4. Condition $\left(C^{\prime}\right)$. Hulanicki [5] proves that $\ell_{1}(G)$ is symmetric for any group $G$ satisfying:

(C) there is a $k$ such that for any finite set $A \subset G$

$$
\sup c\left(A t_{1} A t_{2} \cdots A t_{n}\right) \leqq k^{m} f_{A}(m, n),
$$

where the least upper bound on the left is taken over all sequences $\left(t_{1}, t_{2}, \cdots, t_{n}\right) \in G^{n}$ where at most $m$ of the $t_{i}$ 's are different from the identity, and the function $f_{A}(m, n)$ satisfies the condition $f_{A}(m, n)=$ $o\left(c^{n}\right)$ for any $c>1$, uniformly with respect to $m \leqq n$. We will obtain the same result for any group $G$ satisfying the condition

$\left(C^{\prime}\right)$ there is a $k$ such that for each $A \in \mathscr{S}(G)$

$$
\liminf \sup _{\left(s_{i}\right) \in G^{n}}\left[c\left(A s_{1} A s_{2} \cdots A s_{n}\right)\right]^{1 / n}<k .
$$

Lemma 4.1. If $G$ satisfies $\left(C^{\prime}\right)$ then $G$ also satisfies (SS).

Proof. If $G$ satisfies $\left(C^{\prime}\right)$ then for each $A \in \mathscr{S}(G)$

$$
\liminf { }_{n} c\left(A^{n}\right)^{1 / n}<k .
$$

If for some $B \in \mathscr{S}(G)$,

$$
\lim \inf _{n} c\left(B^{n}\right)^{1 / n} \geqq \delta>1,
$$

then choose a positive integer $p$ so that $\delta^{p} \geqq k$. Then

$$
\lim \inf _{n} c\left(\left(B^{p}\right)^{n}\right)^{1 / n}=\left[\lim \inf _{n} c\left(B^{p n}\right)^{1 / p n}\right]^{p}=\delta^{p} \geqq k .
$$

Thus, for each $A \in \mathscr{S}(G)$,

$$
\lim \inf _{n} c\left(A^{n}\right)^{1 / n} \leqq 1 .
$$

Now, 


$$
c\left(A^{n} \sim A^{n-1}\right) \leqq c\left(A^{n}\right)
$$

for each $n \geqq 2$. Hence

$$
\lim \inf _{n} c\left(A^{n} \sim A^{n-1}\right)^{1 / n} \leqq 1
$$

for each $A \in \mathscr{S}(G)$.

Lemma 4.2. Assume $G$ satisfies $\left(C^{\prime}\right)$ with constant $k$. Let $x$ and $y$ be elements of $\ell_{1}(G)$ such that $N(x)$ is finite. Then

$$
\nu(x y) \leqq k\|y\|\left\|R_{x}\right\| \text {. }
$$

Proof.

$$
\begin{aligned}
\left\|(x y)^{n}\right\| & =\left\|\left(x \sum_{s \in G} y(s) s\right)^{n}\right\|=\left\|\sum_{s_{1}, \cdots, s_{n} \in G} y\left(s_{1}\right) \cdots y\left(s_{n}\right) x s_{1} \cdots x s_{n}\right\| \\
& \leqq \sum_{s_{1}, \cdots, s_{n} \in G}\left|y\left(s_{1}\right)\right| \cdots\left|y\left(s_{n}\right)\right|\left\|x s_{1} \cdots x s_{n}\right\| \\
& \leqq \sup _{s_{1}, \cdots, s_{n} \in G}\left\|x s_{1} \cdots x s_{n}\right\|_{s_{1}, \cdots, s_{n} \in G}\left|y\left(s_{1}\right)\right| \cdots\left|y\left(s_{n}\right)\right| .
\end{aligned}
$$

An application of Schwarz inequality gives

$$
\left\|x s_{1} \cdots x s_{n}\right\| \leqq c\left(N\left(x s_{1} \cdots x s_{n}\right)\right)^{1 / 2}\left\|x s_{1} \cdots x s_{n}\right\|_{2} .
$$

For any $z \in \ell_{1}(G)$

$$
\left\|R_{z}\right\|=\sup _{\|y\|_{2}=1}\left\|R_{z}(y)\right\|_{2} \geqq\left\|R_{z}(e)\right\|_{2}=\|z\|_{2} .
$$

Therefore

$$
\left\|x s_{1} \cdots x s_{n}\right\|_{2} \leqq\left\|R_{x s_{1} \cdots x s_{n}}\right\|=\left\|R_{x s_{1}} R_{x s_{2}} \cdots R_{x s_{n}}\right\| \leqq\left\|R_{x}\right\|^{n} .
$$

Also,

$$
N\left(x s_{1} \cdots x s_{n}\right) \subset N\left(x s_{1}\right) \cdots N\left(x s_{n}\right)=N(x) s_{1} \cdots N(x) s_{n} .
$$

Therefore, if we set $A=N(x) \cup\{e\} \cup N(x)^{-1}$,

$$
\left\|x s_{1} \cdots x s_{n}\right\| \leqq c\left(A s_{1} \cdots A s_{n}\right)^{1 / 2}\left\|R_{x}\right\|^{n} .
$$

Finally

$$
\sum_{s_{1}, \cdots, s_{n} \in G}\left|y\left(s_{1}\right)\right| \cdots\left|y\left(s_{n}\right)\right|=\|y\|^{n} .
$$

Consequently

$$
\left\|(x y)^{n}\right\| \leqq\|y\|^{n}\left\|R_{x}\right\|^{n} \sup _{s_{1}, \cdots, s_{n} \in G} c\left(A s_{1} \cdots A s_{n}\right)^{1 / 2},
$$

and hence 


$$
\begin{aligned}
\nu(x y) & =\liminf _{n}\left\|(x y)^{n}\right\|^{1 / n} \leqq\|y\|\left\|R_{x}\right\| \lim \inf _{n_{s_{1}}, \cdots, s_{n}} \sup _{n} c\left(A s_{1} \cdots A s_{n}\right)^{1 / 2 n} \\
& \leqq k\|y\|\left\|R_{x}\right\| .
\end{aligned}
$$

We are now ready to prove.

THEOREM 4.3. If $G$ satisfies $\left(C^{\prime}\right)$ then $\iota_{1}(G)$ is symmetric.

Proof. If $\ell_{1}(G)$ is not symmetric, then by Raikov's Theorem (c.f. [8], p. 307) there is a $y y^{*} \in \iota_{1}(G)$ such that

$$
r=\sup _{\left.f \in \mathscr{S}_{0} 0^{(*)}\right)} f\left(y y^{*}\right)<\nu\left(y y^{*}\right) \text {. }
$$

We may assume that;

$$
\inf _{f \in \Im_{0}(\mathscr{C})} f\left(y y^{*}\right)=s>0:
$$

if not we consider the element $y y^{*}+\alpha e$ for some $\alpha>0$.

Let $x=y y^{*}$ and choose $u$ and $v$ so that $0<u<s$ and $r<v<\nu(x)$. Then

$$
0<u<f(x)<v<\nu(x)
$$

for each $f \in \mathscr{F}_{0}(\mathscr{H})$.

Let $k$ be the constant of $\left(C^{\prime}\right), t>1$ and $p$ be a positive integer such that $\nu(x)^{p}>k t v^{p}$. Pick $A \in \mathscr{S}(G)$ so that, if $z$ is $x$ restricted to $A$ then

(i) $0<f(z)<v$, for each $f \in \mathscr{F}_{0}(\mathscr{X})$ and

(ii) $\left\|z^{-p} x^{p}\right\|<t$.

To see that (i) is possible merely note that by taking $A$ sufficiently large, $\|z-x\|$ is less than both $v-r$ and $u$. Then, since each $f \in \mathscr{F}_{0}(\mathscr{C})$ is of norm one, the condition is satisfied.

For (ii) we first observe that by Lemma 1.4 and 4.1

$$
\operatorname{sp}(z) \subset\left\{f(z) \mid f \in \mathscr{F}_{0}(\mathscr{C})\right\} \subset(0, v) .
$$

Thus $z$ is regular, and for fixed $p, z^{-p} x^{p}$ converges to the identity as $A$ increases.

We now apply Lemma 4.2.

$$
\nu\left(x^{p}\right)=\nu\left[z^{p}\left(z^{-p} x^{p}\right)\right] \leqq k\left\|z^{-p} x^{p}\right\|\left\|R_{z^{p}}\right\| .
$$

But

$$
\left\|R_{z^{p}}\right\| \leqq\left\|R_{z}\right\|^{p}
$$

and

$$
\left\|R_{z}\right\|=\nu\left(R_{z}\right) \leqq \nu(z)<v
$$

Therefore 


$$
\nu\left(x^{p}\right)=\nu(x)^{p} \leqq k t v^{p} .
$$

But $p$ was chosen so that

$$
\nu\left(x^{p}\right)>k t v^{p} .
$$

This contradiction implies that $\ell_{1}(G)$ is symmetric.

5. Nonsymmetric group algebras. In [3], Frey asked if there are amenable groups with nonamenable subsemigroups. He proved that if such groups exist they must contain a free nonabelian subsemigroup on two generators. Hochster [4], has recently presented an example of such a group. In [7], a similar example is presented, and it is shown that the algebra of this group is nonsymmetric. The following theorem shows that all such groups have nonsymmetric algebras. The proof employs the well known fact that in a symmetric Banach *-algebra the hermitian elements have real spectra (c.f. [8]).

THEOREM 5.1. Let $G$ be a group generated by $a$ and $b$ such that $S$, the semigroup generated by $a$ and $b$ is free. Then, $\ell_{1}(G)$ is nonsymmetric.

Proof. We will show that $\delta i \in \mathrm{sp}(x)$, where

$$
x=\alpha a+\beta b+\lambda a b+\bar{\lambda} b^{-1} a^{-1}+\bar{\beta} b^{-1}+\bar{\alpha} a^{-1},
$$

if $\delta i=\alpha \beta / \lambda$ and $|\lambda| \geqq \max \{3|\alpha|, 3|\beta|\}$. To accomplish this we will construct a nonzero $\theta \in m(G)$ such that $\theta^{v}$ vanishes on the left ideal generated by $y=x-\delta i e$.

Let $S_{0}=S \cup\{e\}$ and $S_{1}=a S_{0} \cup\{e\} \cup b^{-1} S_{0}^{-1}$. Define $\theta(g)=0$ if $g \notin S_{1}$. Let $A=N(y)$ and $S^{\prime}=S_{0} \cup b^{-1} S_{0} \cup S_{0}^{-} \cup a S_{0}^{-1}$. Direct computations yield:

$$
A g \cap S_{1} \neq \varnothing \Leftrightarrow g \in S^{\prime} .
$$

Enumerate the elements of $S^{1}$ as follows: $s_{1}=e$ and for $n=$ $1,2, \cdots, s_{2 n}=a s_{n} ; s_{2 n+1}=b s_{n} ; t_{n}=b^{-1} s_{2 n}: s_{-1}=s_{1}$ and for $n=1,2, \cdots$, $s_{-2 n}=a^{-1} s_{-n} ; s_{-(2 n+1)}=b^{-1} s_{-n} ; t_{-n}=a s_{-(2 n+1)}$.

One can easily verify that the homogeneous equations $\theta^{\circ}\left(y s_{i}\right)=$ $0,-3 \leqq i \leqq 2, i \neq 0$, and $\theta^{v}\left(y t_{j}\right)=0,-2 \leqq j \leqq 2, j \neq 0$, have a nontrivial simultaneous solution.

For $n$ a positive integer,

$$
N\left(y t_{n}\right)=\left\{s_{2 n}, s_{4 n}, a t_{n}, a^{-1} t_{n}, b^{-1} a^{-1} t_{n}, b^{-1} t_{n}, t_{n}\right\} .
$$

If $a t_{n}=a b^{-1} s_{2 n} \in a S_{0}$ then $b^{-1} s_{2 n} \in S_{0}$ which is impossible. Similarly, if $a t_{n} \in b^{-1} S_{0}^{-1}$ then $b^{-1}=a^{-1} s^{-1}$ for some $s \in S$. Since $S$ is free in $G$, 
this also is impossible. Certainly $a t_{n} \neq e$. Thus $a t_{n} \notin S_{1}$. Similar arguments show that

$$
\left\{a^{-1} t_{n}, b^{-1} a^{-1} t_{n}, a^{-1} a^{-1} t_{n}, t_{n}\right\} \cap S_{1}=\varnothing .
$$

We also have

$$
N\left(y s_{n}\right)=\left\{s_{2 n}, s_{2 n+1}, s_{4 n+2}, s_{n}, a^{-1} s_{n}, b^{-1} s_{n}, b^{-1} a^{-1} s_{n}\right\} .
$$

For $n \geqq 3$,

$$
N\left(y s_{n}\right) \cap S_{1}=N\left(y s_{n}\right) \cap\left(a S_{0} \cup\{e\}\right) .
$$

If $n$ is odd then

$$
N\left(y s_{n}\right) \cap S_{1} \subset\left\{s_{2 n}, s_{4 n+2}, b^{-1} s_{n}\right\}
$$

while for $n$ even

$$
N\left(y s_{n}\right) \cap S_{1} \subset\left\{s_{2 n}, s_{4 n+2}, a^{-1} s_{n}, b^{-1} a^{-1} s_{n}, s_{n}\right\} .
$$

Note that not both $a^{-1} s_{n}$ and $b^{-1} a^{-1} s_{n}$ are elements of $S_{1}$. Also, $a^{-1} s_{n}=s_{m} \in S_{1}$ implies $m<n$ and $b^{-1} a^{-1} s_{n}=s_{m} \in S_{1}$ implies $m<n$.

Assume now that $n \geqq 3$ is given, and that $\theta\left(s_{k}\right)$ has been defined for $1 \leqq k<4 n$ such that $\left|\theta\left(s_{k}\right)\right| \leqq 1$ and

$$
\theta^{v}\left(y t_{m}\right)=0=\theta^{v}\left(y s_{m}\right)
$$

for $m<n$. Now

$$
\theta^{v}\left(y t_{n}\right)=\beta \theta\left(s_{2 n}\right)+\lambda \theta\left(s_{4 n}\right)
$$

and

$$
\begin{aligned}
\theta^{v}\left(y s_{n}\right)=\alpha \theta\left(s_{2 n}\right) & +\lambda \theta\left(s_{4 n+2}\right)+\bar{\alpha} \theta\left(a^{-1} s_{n}\right)+\bar{\beta} \theta\left(b^{-1} s_{n}\right)+\bar{\lambda} \theta\left(b^{-1} a^{-1} s_{n}\right) \\
& +\delta i \theta\left(s_{n}\right) .
\end{aligned}
$$

Let

$$
\theta\left(s_{4 n}\right)=(-\beta / \lambda) \theta\left(s_{2 n}\right)
$$

and

$$
\begin{aligned}
\theta\left(s_{4 n+2}\right)= & (-\bar{\lambda} / \lambda) \theta\left(b^{-1} a^{-1} s_{n}\right) \\
& -1 / \lambda\left[\alpha \theta\left(s_{2 n}\right)+\bar{\alpha} \theta\left(a^{-1} s_{n}\right)+\bar{\beta} \theta\left(b^{-1} s_{n}\right)+\delta s \theta\left(s_{n}\right)\right] .
\end{aligned}
$$

We consider the two possibilities:

(i) If $b^{-1} a^{-1} s_{n} \in S_{1}$ then $b^{-1} a^{-1} s_{n} \in a S_{0} \cup\{e\}$ and hence

$$
\left\{a^{-1} s_{n}, b^{-1} s_{n}\right\} \cap S_{1}=\varnothing \text {. }
$$

Also, there is an $n>m \geqq 1$ such that $s_{n}=s_{2 m}$. Since $\theta^{v}\left(y t_{k}\right)=0$ 
for $1<k<n$ and since $\theta^{v}\left(y t_{k}\right)=\beta \theta\left(s_{2 k}\right)+\lambda \theta\left(s_{4 k}\right)=0$ if, and only it. $\theta\left(s_{4 k}\right)=(-\beta / \lambda) \theta\left(s_{2 k}\right) ; \theta\left(s_{2 n}\right)=\theta\left(s_{4 m}\right)=(-\beta / \lambda) \theta\left(s_{2 m}\right)$. Thus

$$
\begin{aligned}
\theta\left(s_{4 n+2}\right) & =(-\bar{\lambda} / \lambda) \theta\left(b^{-1} a^{-1} s_{n}\right)-(1 / \lambda)(\delta i-\alpha \beta / \lambda) \theta\left(s_{2 m}\right) \\
& =(-\bar{\lambda} / \lambda) \theta\left(b^{-1} a^{-1} s_{n}\right) .
\end{aligned}
$$

(ii) If $b^{-1} a^{-1} s_{n} \notin S$, then

$$
\theta\left(s_{4 n+2}\right)=(-1 / \lambda)\left[\alpha \theta\left(s_{2 n}\right)+\bar{\alpha} \theta\left(a^{-1} s_{n}\right)+\bar{\beta} \theta\left(b^{-1} s_{n}\right)+\delta i \theta\left(s_{n}\right)\right]
$$

and at most three terms within the parenthesis on the right are nonzero.

In either case $\left|\theta\left(s_{4 n+2}\right)\right| \leqq 1$. Certainly $\left|\theta\left(s_{4 n}\right)\right| \leqq 1$. Thus, by induction, we can define $\theta\left(s_{2 n}\right)$ such that $\left|\theta\left(s_{2 n}\right)\right| \leqq 1$ and such that

$$
\theta^{v}\left(y t_{n}\right)=0=\theta^{v}\left(y s_{n}\right)
$$

for $n=1,2, \cdots$.

Similarly, we can define $\theta\left(s_{-(2 n+1)}\right)$ so that $\left|\theta\left(s_{-(2 n+1)}\right)\right| \leqq 1$ and

$$
\theta^{v}\left(y t_{-n}\right)=0=\theta^{v}\left(y s_{-n}\right)
$$

for $n=1,2, \cdots$.

REMARK. If $G$ is an amenable group with a nonamenable subsemigroup then $G$ has a subgroup $H$ that satisfies the hypothesis of Theorem 5.1. Hence $\ell_{1}(H)$ is nonsymmetric, and since for each $x \in \ell_{1}(H)$,

$$
s p_{\ell_{1}(H)}(x)=\operatorname{sp}_{\ell_{1}(G)}(x)
$$

$\ell_{1}(G)$ is also nonsymmetric.

\section{REFERENCES}

1. G. M. Adel 'son-Vel' skil and Yu A. Šreider, The Banach mean on groups, Uspeh, Mat. Nauk (N. S.) 12 (1957), 131-136.

2. R. A. Bonic, Symmetry in group algebras of discrete groups, Pacific J. Math. 11 (1961), 73-94.

3. A. H. Frey, Studies in amenable semigroups, Thesis, University of Washington, Seattle, Washington, 1960.

4. M. Hochster, Subsemigroups of amenable groups (to appear)

5. A. Hulanicki, On the spectral radius of hermitian elements in group algebras, Pacific J. Math. 18 (1966), 277-290.

6. A. Hulanicki, Groups whose regular representations weakly contain all unitary representations, Studia Math. 24 (1964), 37-59.

7. J. W. Jenkins, An amenable group with a non-symmetric group algebra. Bull. Amer. Math. Soc. 75 (1969), 357-360.

8. M. A. Naimark, Normed rings, P. Noordhoff, Ltd., Groningen, 1960. 
Received February 3, 1969. Some of the results stated in this paper are found in the author's doctoral dissertation, written at the University of Illinois under the direction of Professor M. M. Day. Partial support was received from National Science Foundation Research Grant GP-6990.

State University of New York at Albany 



\section{PACIFIC JOURNAL OF MATHEMATICS}

\section{EDITORS}

\author{
H. SAMELSON \\ Stanford University \\ Stanford, California 94305 \\ Richard Pierce \\ University of Washington \\ Seattle, Washington 98105
}

J. DUGUNDJI

Department of Mathematics

University of Southern California

Los Angeles, California 90007

BASIL GORDON*

University of California

Los Angeles, California 90024

\section{ASSOCIATE EDITORS}
E. F. BECKENBACH
B. H. NeUmanN
F. WOLF
K. YoSHIDA

\section{SUPPORTING INSTITUTIONS}

UNIVERSITY OF BRITISH COLUMBIA CALIFORNIA INSTITUTE OF TECHNOLOGY

UNIVERSITY OF CALIFORNIA MONTANA STATE UNIVERSITY

UNIVERSITY OF NEVADA

NEW MEXICO STATE UNIVERSITY

OREGON STATE UNIVERSITY

UNIVERSITY OF OREGON

OSAKA UNIVERSITY

UNIVERSITY OF SOUTHERN CALIFORNIA
STANFORD UNIVERSITY

UNIVERSITY OF TOKYO

UNIVERSITY OF UTAH

WASHINGTON STATE UNIVERSITY

UNIVERSITY OF WASHINGTON

AMERICAN MATHEMATICAL SOCIETY CHEVRON RESEARCH CORPORATION TRW SYSTEMS

NAVAL WEAPONS CENTER 


\section{Pacific Journal of Mathematics}

\section{Vol. 32, No. $1 \quad$ January, 1970}

Robert Alexander Adams, Compact Sobolev imbeddings for unbounded domains ........................................ 1

Bernhard Amberg, Groups with maximum conditions .................. 9

Tom M. (Mike) Apostol, Möbius functions of order k............... 21

Stefan Bergman, On an initial value problem in the theory of two-dimensional transonic flow patterns ................... 29

Geoffrey David Downs Creede, Concerning semi-stratifiable spaces ...... 47

Edmond Dale Dixon, Matric polynomials which are higher

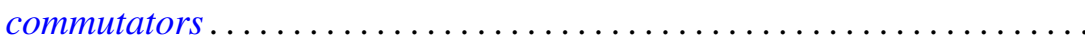

R. L. Duncan, Some continuity properties of the Schnirelmann density.

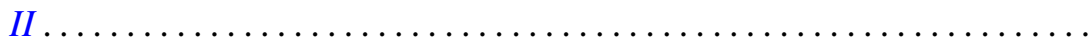

Peter Larkin Duren and Allen Lowell Shields, Coefficient multipliers of $H^{p}$

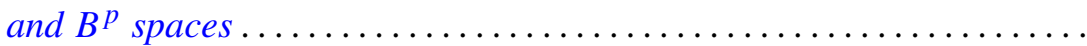

Hector O. Fattorini, On a class of differential equations for vector-valued

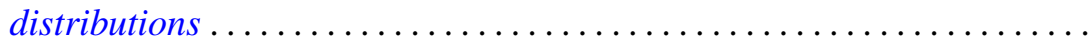

Charles Hallahan, Stability theorems for Lie algebras of derivations. . . . . . 105

Heinz Helfenstein, Local isometries of flat tori ................ 113

Gerald J. Janusz, Some remarks on Clifford's theorem and the Schur

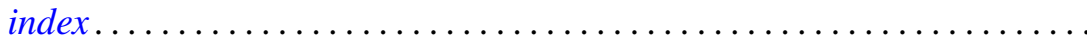

Joe W. Jenkins, Symmetry and nonsymmetry in the group algebras of discrete groups. ...

Herbert Frederick Kreimer, Jr., Outer Galois theory for separable

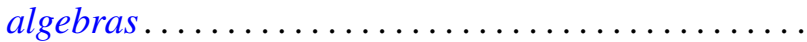

D. G. Larman and P. Mani, On visual hulls

R. Robert Laxton, On groups of linear recurrences. II. Elements of finite order.

Dong Hoon Lee, The adjoint group of Lie groups ...

James B. Lucke, Commutativity in locally compact rings

Charles Harris Scanlon, Rings of functions with certain Lipschitz

$$
\text { properties ............................... }
$$

Binyamin Schwarz, Totally positive differential systems .

James McLean Sloss, The bending of space curves into piecewise helical curves.

James D. Stafney, Analytic interpolation of certain multiplier spaces ...

Patrick Noble Stewart, Semi-simple radical classes.......

Hiroyuki Tachikawa, On left $\mathrm{QF}-3$ rings ...................... 255

Glenn Francis Webb, Product integral representation of time dependent nonlinear evolution equations in Banach spaces.... . . . 